\title{
Réplique au compte rendu de Chantal Legault sur Les textes poétiques du Canada français, 1606-1867. Édition intégrale, volume $4: 1838-1849$, par Yolande GRISÉ et Jeanne-d'Arc LORTIE, s.c.o., avec la collaboration de Pierre SAVARD et Paul WYCZYNSKI (Montréal, Éditions Fides, 1991), paru dans le volume 46, numéro 2 (automne 1992) : 320-322.
}

Yolande Grisé

Volume 47, numéro 3, hiver 1994

URI : https://id.erudit.org/iderudit/305274ar

DOI : https://doi.org/10.7202/305274ar

Aller au sommaire du numéro

Éditeur(s)

Institut d'histoire de l'Amérique française

ISSN

0035-2357 (imprimé)

1492-1383 (numérique)

Découvrir la revue

Citer ce document

Grisé, Y. (1994). Réplique au compte rendu de Chantal Legault sur Les textes poétiques du Canada français, 1606-1867. Édition intégrale, volume 4 : 1838-1849, par Yolande GRISÉ et Jeanne-d'Arc LORTIE, s.c.o., avec la

collaboration de Pierre SAVARD et Paul WYCZYNSKI (Montréal, Éditions Fides, 1991), paru dans le volume 46, numéro 2 (automne 1992) : 320-322. Revue d'histoire de l'Amérique française, 47(3), 465-468.

https://doi.org/10.7202/305274ar d'utilisation que vous pouvez consulter en ligne. 


\section{RÉPLIQUE}

Réplique de Yolande Grisé au compte rendu de Chantal Legault sur Les textes poétiques du Canada français, 1606-1867. Édition intégrale, volume 4: 1838-1849, par Yolande GRISÉ et Jeanne-d'Arc LORTIE, s.c.o., avec la collaboration de Pierre SAVARD et Paul WYCZYNSKI (Montréal, Éditions Fides, 1991), paru dans le volume 46, numéro 2 (automne 1992): 320-322.

Le numéro d'automne 1992 de la Revue d'histoire de l'Amérique française (p. 320-322) présente un compte rendu de l'ouvrage mentionné cidessus, qui révèle un intérêt certain pour nos travaux et une connaissance approfondie de la période recensée dans ce volume. Peu de critiques ont cerné d'aussi près le dessein de cette large entreprise et, en même temps, y ont apporté des commentaires aussi circonstanciés. Rappelons qu'il s'agit de la publication intégrale, en 12 volumes, d'un corpus de quelque 3500 textes réunissant environ 220000 vers.

Dans ce compte rendu, Chantal Legault déplore «certaines lacunes» au niveau de la recherche. Nous lui savons gré de nous signaler des pièces qui auraient dû apparaître dans ce volume ou dans les volumes précédents. Conscients qu'une quête de cette envergure, aussi considérablement étalée dans le temps et ayant pour but de rassembler la totalité d'une production imprimée, ne saurait être définitive, nous avons donc prévu, dès le début des travaux, publier en fin de parcours, sous forme de Supplément, les textes retrouvés ou signalés en cours de route, mais n'ayant pu figurer à l'endroit approprié dans la série. Cette précision sera mentionnée dans les prochains volumes à paraître, afin de rassurer les lecteurs sur ce point.

Certaines attributions de madame Legault peuvent cependant être contestées: Marie-Olympe Carpentier, couturière de La Flèche et auteure du poème intitulé «L'Hirondelle» dans la livraison du 22 juin 1839 de L'Ami du Peuple, de l'Ordre et des Lois, a toutes les chances de n'être pas Canadienne, mais bien Française... de France. À notre connaissance, il n'y a pas de localité du nom de La Flèche au Bas-Canada. Il est déraisonnable de suggérer que le poème ait pu être écarté parce que son auteur était une femme, ou encore une travailleuse. L'usage des pseudonymes de même que la présence de nombreux textes anonymes nous eût interdit de procéder avec certitude à de pareilles exclusions, si une telle méthode avait été notre façon de travailler. D'autre part, même dans le cas où il se serait agi d'un pseudonyme, nous aurions volontiers ajouté ce nom à la quinzaine de noms féminins inclus dans les quatre premiers volumes de la collection.

RHAF, vol. $47, \mathrm{n}^{\circ} 3$, hiver 1994 
Même remarque au sujet de William Jouvante, jeune garçon de douze ans, qui a signé dans Le Canadien du 17 mai 1843 un poème intitulé «À ma soeur mourante». À notre connaissance, rien ne permet de repérer l'individu dans la vallée du Saint-Laurent à cette époque. Ce n'est certes pas l'âge du poète qui est en cause ici: nous n'avons pas écarté le texte intitulé «Vers d'un enfant à son chien» et attribué à André-Romuald Cherrier alors qu'il n'était âgé que de «treize ans et huit mois» (vol. 3, $\mathrm{n}^{\circ}$ 239). Au surplus, plusieurs textes rimés retenus sont attribués à des écoliers, à des élèves ou à des étudiants de collèges ou du Petit Séminaire de Québec. Tout spécialiste de la recherche aura conclu que seul un doute raisonnable sur l'identité des auteurs ou sur la provenance des textes nous a incités à faire preuve de prudence en matière d'attribution de textes.

On aura compris du reste que, par sa nature même, la collection favorise précisément la connaissance des "petits poètes» (poetae minores) des années 1606-1867 et la diffusion de leurs vers, condamnés autrement à l'oubli. Ainsi, la collection restitue aux héritiers de ce patrimoine culturel, en même temps que leur littérature des origines, l'origine de leur littérature: une langue française riche d'images, de musiques, de cadences, de tout un héritage littéraire savant et populaire. Porteurs d'une culture vivante, les poètes d'autrefois, des plus connus aux plus obscurs, ont contribué, à leur manière, à la rendre vivace.

Plus sérieuses nous paraissent les prétendues "graves déficiences» soulignées par Chantal Legault au plan des variantes. Une lecture attentive de l'introduction du premier volume de la collection, où sont exposées «Les Règles de présentation des textes» (p. lxvi, section $C, 1, c)$, lui aurait permis de prendre connaissance de l'avertissement suivant: "A noter cependant que nous ne donnons que les principales variantes, et que pour de rares poèmes, lorsqu'elles présentent un intérêt particulier pour l'évolution de la langue, la graphie populaire et l'effort créateur de l'auteur.» Une telle précision ne figure pas dans les autres volumes de la collection, mais peut-être le devraitelle, afin de le rappeler aux lecteurs.

Quoi qu'il en soit, toutes les références des pièces parues avant 1867 figurent dans l'abondante bibliographie qui précède le corpus. Le chercheur curieux de connaître les différentes versions d'un poème bénéficie donc de renseignements qui facilitent le repérage du texte. Au surplus, afin d'éclairer rapidement le chercheur sur l'ampleur des changements opérés dans chacune de ces versions, nous lui fournissons certaines indications utiles. On aura saisi, en lisant attentivement cette introduction, que l'objectif de la collection n'est pas la publication de toutes les variantes d'un texte, mais bel et bien l'édition intégrale de sa première version imprimée (vol. 4, p. xxii). On ne peut donc raisonnablement mettre au compte d' «une grave déficience» scientifique ce qui n'est pas l'objectif du projet. Ajoutons que, dès que la collection aura été publiée dans son entier, la documentation accumulée au cours de ce vaste dépouillement sera confiée aux soins du Service des archives du Centre de recherche en civilisation canadienne-française de l'Université d'Ottawa et, de ce fait, rendue accessible à tous les chercheurs. 
En attendant ce jour, grâce aux références fournies dans la bibliographie, les chercheurs intéressés peuvent aisément retrouver les versions qui les intéressent dans les bibliothèques ou les services d'archives où elles sont conservées.

Lorsque nous affirmons dans l'avant-propos du premier volume de la collection (p. xiii), auquel Chantal Legault se réfère, offrir au lecteur un corpus de textes "présentés et annotés avec rigueur», nous ne faisons nullement allusion aux variantes: nous nous référons explicitement à l'introduction de même qu'aux notices historiques et aux notes explicatives qui accompagnent les textes des deux premiers volumes de la collection. Malheureusement, à partir du troisième volume, il nous a fallu renoncer à la dimension explicative détaillée de nos travaux, sous la contrainte d'impératifs indépendants de notre volonté, tel que nous nous en expliquons dans l'introduction de ce volume. Cependant, on reconnaîtra que cela ne met nullement en cause la rigueur de notre méthode quant à l'établissement de la première publication des textes poétiques, objectif fondamental de la collection.

Au sujet du reproche concernant l'omission totale ou partielle de la publication des textes en prose dont nous faisons parfois précéder les poèmes, nulle part nous n'affirmons nous engager à «reproduire en entier» ces textes en prose. Nous le répétons: dans cette collection, il s'agit essentiellement de donner à lire aux lecteurs d'aujourd'hui et de demain la première publication des textes poétiques du Canada français. C'est par unique souci de reconstituer rapidement le contexte d'une pièce que nous restituons, au besoin, quelques lignes ou extraits en prose susceptibles d'éclairer le lecteur. Les contraintes d'espace et de coût de publication nous empêchent de reproduire tous les textes en prose - souvent de grand intérêt - qui accompagnent certains poèmes. Nous avons choisi plutôt de doter les chercheurs d'un outil bien documenté afin de leur faciliter la poursuite de recherches auxquelles nous ne pouvions nous attarder sans déroger à l'objectif central du projet. Nous sommes persuadés que d'autres chercheurs viendront après nous, qui trouveront leur bien dans cet immense chantier du discours versifié que nous avons consciencieusement défriché, avec peu de moyens. Entre temps, nous poursuivons patiemment notre tâche, stimulés en cela par le poète latin Virgile, malheureux que Rome eût conservé si peu de traces d'Ennius et des poètes anciens.

L'édition intégrale des Textes poétiques du Canada français, 1606-1867 n'est certes pas «l'instrument rêvé des spécialistes» peu expérimentés dans les travaux de longue haleine ou rebutés par l'effort. C'est un outil modeste et authentique. Nous le croyons utile aussi à une meilleure connaissance des aspirations et des réalisations, des revers et des rêves du Canada français et du Québec. Nous souhaitons qu'il soit un levier capable de soulever les montagnes d'ignorance, d'amnésie, d'indifférence ou de préjugés qui recouvrent nos origines littéraires, fondements de la culture canadienne-française et québécoise. Nous croyons que l'étude et l'enseignement de ces textes susciteront, surtout chez les jeunes, l'émergence d'une prise de conscience de la valeur de cet héritage commun. Nous sommes convaincus de la nécessité 
de bien connaître le passé pour envisager l'avenir et s'y mesurer, particulièrement en des temps d'incertitude et d'insécurité, refrains bien connus de nos aïeux.

Université d'Ottawa

YOLANDE GRISÉ directrice du projet 